\title{
Thermal Management Concepts for Vehicles of Next Generation
}

\author{
Michael Schier, Nina Kevlishvili, Bastian Mayer \\ Institute of Vehicle Concepts \\ German Aerospace Center \\ Pfaffenwaldring 38-40 \\ D-70569 Stuttgart \\ Email: michael.schier@dlr.de, nina.kevlishvili@dlr.de, \\ bastian.mayer@dlr.de
}

\begin{abstract}
The present paper is a review of the selected research activities at the German Aerospace Center on the field of terrestrial vehicles thermal management. The methods and some results of the passenger cabin measurements and human comfort studies are described. Several shortly depicted concepts for the cabin airconditioning present the challenges of current and future research topics. Some examples of newly developed highly integrated components and the component tempering strategies for next generation vehicles are given.
\end{abstract}

Keywords-next generation car; thermal management; active expansion; magnetocaloric refrigeration; fuel cells; thermochemical storage; thermoelectric generator.

\section{INTRODUCTION}

In conventional vehicles the amount of residual heat is largely sufficient to cover all air-conditioning needs. Even more, a part of the heat waste of the combustion motor can be converted to the electric voltage by means of thermoelectric generator to extend the range of conventional or hybrid vehicle [1]. With the increasing electrification of vehicles, heating and cooling become the main challenges. There is no natural source of heat but in addition to the cabin climatisation, it is necessary to provide the energy for the safe operation of temperature dependent components. The drive train cannot be considered as the single source of energy for the thermal management. The obvious possibility is then, to access the energy of first source, the battery, for example. But this immediately leads to decrease of the vehicle's range. Furthermore, the operation of some sensitive components, e.g. fuel-cells or life durability of electrochemical energy (battery), explicitly depends on the temperature in their surroundings. In case of very warm or very cold ambient temperatures the effort to temper these specific components from the same first energy source leads to the further reduction of the range.

In the light of such growing challenges one has to consider the thermal management of the vehicle as an entire, analyze the system requirements and design the solution for the particular needs. As a consequence the development of Vehicles of the Next Generation takes the combination of different technical disciplines into account. There is a large interaction between the vehicle concept by itself, the vehicle structure issues, components of the drive train and chassis as well as the vehicle intelligence and not least the feeling of well-being of passengers.

The thermal management is one of the focal research points at the German Aerospace Center on the field of terrestrial vehicles. The activities build on the highly detailed analysis and characterization of the vehiclespecific energy demand. As it will be shown in this paper, we apply the thermography for the study of heat losses in the vehicles.

In the passenger compartment mockup we investigate air flow, temperature distribution and comfort feeling creating the realistic conditions. Our methods allow us precise simulations and three dimensional visualizations. Here it is a benefit to use synergies from space and aeronautics research, where cabin climatisation is applied to aircraft cabins.

The results of exact measurements are applied for the target-oriented application of existing thermal equipment and implementation of new concepts. Here we will sketch two new concepts of air-conditioning, active expansion and magnetocaloric refrigeration. On the pure component level we present some innovative thermal energy storages and converters and highly integrated concept merging the drive motor and air compressor. 


\section{CURRENT RESEARCH TOPICS}

\section{A. Comfort}

To reduce the energy consumption, it is necessary to study the cabin insulation by means of new composite materials and sandwich structures, or usage of electrooptical panels. Figure 1 shows the thermography of a passenger car to study the heat losses through the windows, through seals and openings as well as the thermal insulation by the interior parts in the doors and in the roof structure. Simultaneous the temperatures in the cabin are recorded to get the thermal time constant as well as local temperatures within the cabin. The measured values are used to calibrate the simulation models.

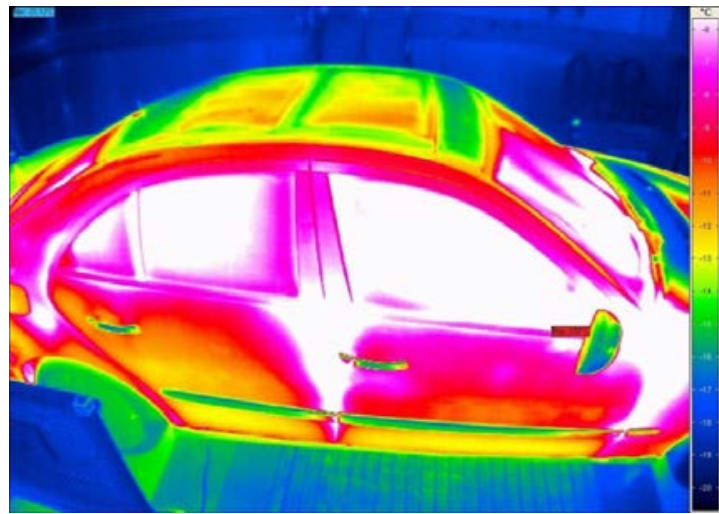

Fig. 1: Thermography of a passenger car interior

Furthermore, we continue to use methods from aeronautics and space technologies [1] to register and control the air quality and develop new ventilation concepts for the cabin under consideration of the amended external aerodynamics. Among other we investigate the well-being of passengers in the cabin by using the vehicle cabin mockups (figure 3) and adopting the test setups for aircraft cabin to terrestrial (rail and road) vehicles. The cabin mockup shown in figure 3 allows the investigation of the energy demand for different air inlet concepts. The flow behavior of the air is also made visible with a multi three-dimensional laser layer method, figure 2.

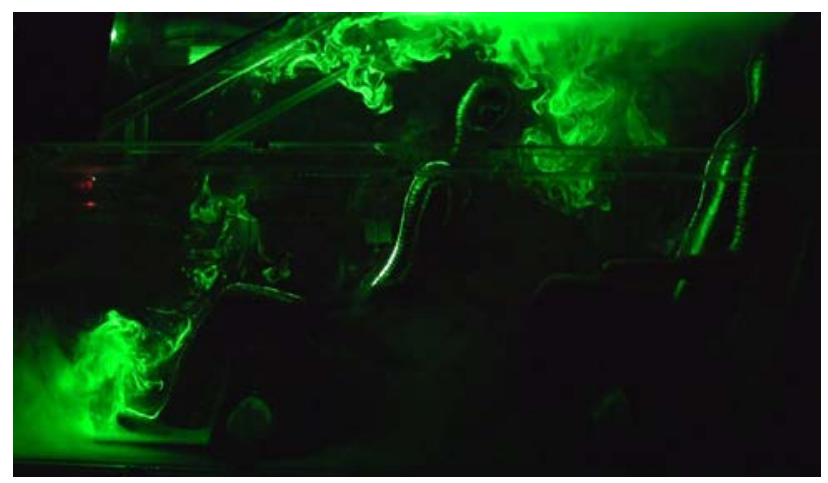

Fig. 2: Laser visualized air flow in the cabin mockup

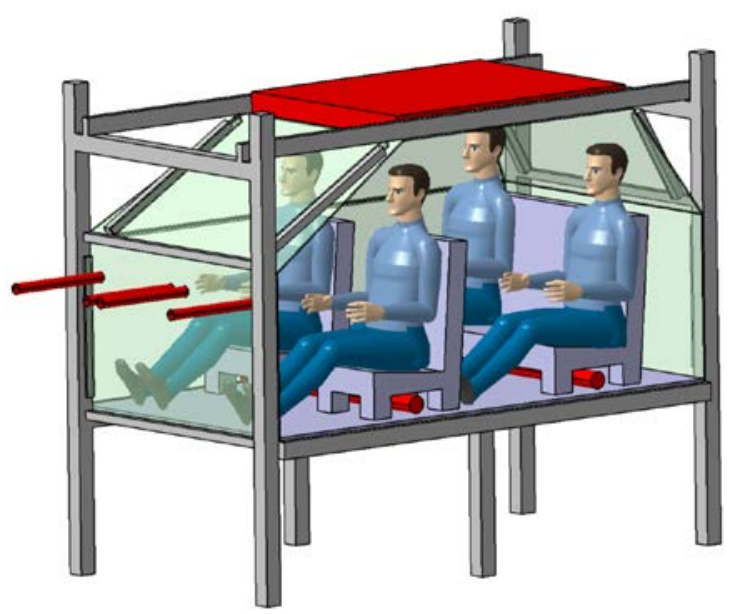

Fig. 3: Cabin mock up

\section{B. Active Expansion}

An environmental friendly approach for vaporcompression refrigeration is the use of $\mathrm{CO}_{2}$ as refrigerant. Compared to fluoride based refrigerants as R1234yf, $\mathrm{CO}_{2}$ provides a four times lower global warming potential (GWP). The disadvantages are the much higher pressure levels which is about five times higher compared to fluoride based refrigerant and the decreased efficiency. The critical point of $\mathrm{CO}_{2}$ is at $31^{\circ} \mathrm{C}$ and 74 bar, which means that the typical condenser temperatures are in the transcritical area and thus are called gas coolers instead of condensers. To increase the efficiency it was recommended by $[6,7]$ to use the energy of the expansion to drive the compression (figure 4). This could be done by transferring the knowledge of a free piston linear generator as it was developed at the institute to build a compressorexpansion unit $[1,3,4]$.

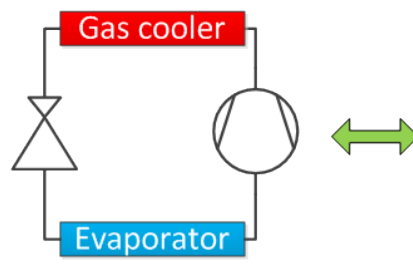<smiles>CC1CC2CCC(CC2)C1</smiles>

Compressor

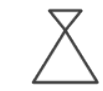

Expansion valve
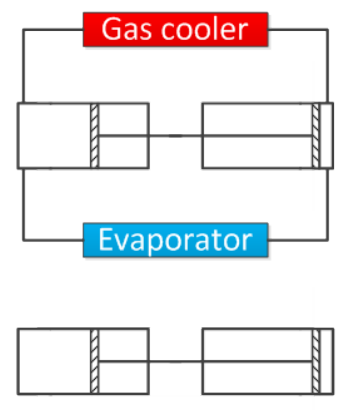

Compressor/ Expansion unit
Fig. 4: Schema of the simple vapour compression cycle

In this compressor-expansion unit depicted in figure 4 the compressor and the expansion valve, which are part of every vapor compression system, are replaced by a device which is similar to a free piston linear generator. The two 
pistons are connected by a single rod and a linear motor is placed in the middle of the unit. The two cylinder tubes work as compression (right) and expansion (left) chambers. The expanding transcritical gas compresses the subcritical gas in the compression chamber to a higher pressure as long as its pressure is higher than in the compression chamber. Further expansion and compression is realized by activating the linear motor which puts a force on the rod.
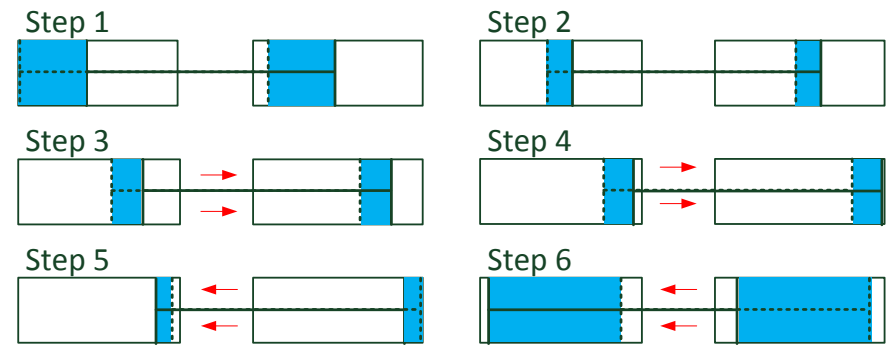

Fig. 5: Active Expansion process

The process can be divided into six steps (see figure 5). During the first step the transcritical gas of the condenser flows into the expansion chamber and compresses the subcritical gas of the right chamber, as it is on a lower pressure level. In step two the valve of the expansion chamber is closed and the expanding gas is driving the piston further into the compression chamber until the pressure in both chambers is equal. With the additional force of the linear generator the gas is further compressed to the needed gas cooler pressure in step three. While the pressure of the transcritical gas in the expansion chamber decreases it cools down and enters the two-phase-region. By reaching the gas cooler pressure the valve on the compression chamber opens (step four) and the piston pushes the transcritical gas out of the compression chamber, the gas/liquid mixture of the expansion chamber is overexpanded due to the large differences in density. The overexpanded mixture is compressed to the needed evaporator pressure in step five while the compression chamber is filled with subcritical gas. At the end of step six the evaporation chamber is empty while the compression chamber valve is closed and the process starts again.

\section{Magnetocaloric refrigration}

On the way to the environmental friendly, efficient and affordable air-conditioning system for the vehicles a great deal of interest is in using the refrigerators based on the magnetocaloric effect. The magnetocaloric effect uses the property of certain materials to heat up in a magnetic field and cool down again reversible upon removal of the magnetic field. When the magnetic field is applied, the unpaired spins align along the magnetic field. This decreases the entropy of the sample in the isotropic process and warms up the system in the adiabatic process. When the magnetic field is switched off the spins randomize increasing the entropy of the sample and the system cools.

According to the latest results of material research the giant magnetocaloric effect (GMCE) near the room temperature is found in the hundreds of Gadolinium (Gd) alloys $G d_{5}\left(S i_{1-x} G e_{x}\right)$. The other families of compounds and alloys which have at least one composition with a GMCE near room temperature include the rare earth manganites $\left(R_{1-x} M_{\chi}\right) M n O_{3}$, where $R$ stays for a rare earth metal and $M$ for $\mathrm{Ca}$, $\mathrm{Sr}$ or $\mathrm{Ba}$; $\mathrm{Mn}\left(\mathrm{As}_{1-\mathrm{x}} \mathrm{S} b_{x}\right)$ and $\mathrm{MnFe}\left(\mathrm{P}_{1-\chi} A s\right)$ alloys; Heusler alloys $N i_{\sim 2} M n_{\sim 1} G e_{\sim 1} ; \quad L a\left(F_{13-x} S i_{x}\right)$ and $\mathrm{La}\left(\mathrm{Fe}_{13-x} \mathrm{Si}_{x}\right) \mathrm{H}_{y}$ alloys [8, 9].

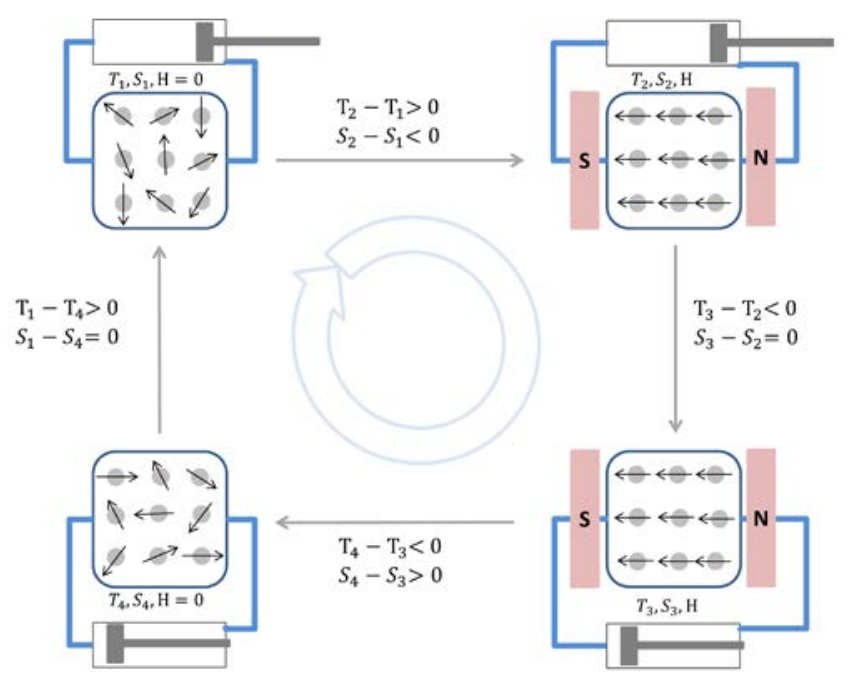

Fig. 6: Schematic illustration of the AMR cycle

In the figure 6 the most efficient cycle for the near room temperature applications, the active magnetic regenerator (AMR) cycle [10] is depicted. The AMR cycle experiences four processes:

a. Magnetization process causing heating of the magnetocaloric material. The heated material rejects heat to the heat transfer fluid in the regenerator increasing its temperature;

b. Cooling process of the regenerator at constant magnetic field by means of displacement of heat transfer fluid from the regenerator with fluid coming from the cold source of the heat exchanger. The fluid absorbs heat from the regenerator and releases it at higher temperature than that of the hot source in the heat exchanger;

c. Demagnetization process. The magnetic field is switched off causing the cooling of the regenerator 
material. It absorbs the heat from the heat transfer fluid and lowers its temperature below the temperature of the cold source;

d. Heating process at zero magnetic field. The heat transfer fluid becomes displaced from the regenerator to the cold source heat exchanger by the fluid from the hot source heat exchanger. The fluid in the cold source heat exchanger absorbs heat, this completes the cycle.

Theoretically, the heat pumps based on the magnetocaloric effect can double the performance of a conventional chiller. The development and integration of the above described system in the road and rail vehicles is the issue of the current research. A COP up to 5 is expected.

\section{Highly integrated Function Concepts}

One specific aspect of thermal management is the temperature control of the drive train components, particularly in the integration of several functions of subcomponents in one component, for example, in the battery or in the electric motor. Examples of highly integrated motors for aircraft application have already been shown [1]. Particular attention is still in the contemplation of the drive motor and its connection to the air conditioning system of the vehicle. To optimize the heat dissipation, novel cooling methods are of great interest. For the integration of additional drive train components in the drive motor, such as the gearbox, a common oil cooling circuit for the two subcomponents is of high interest.

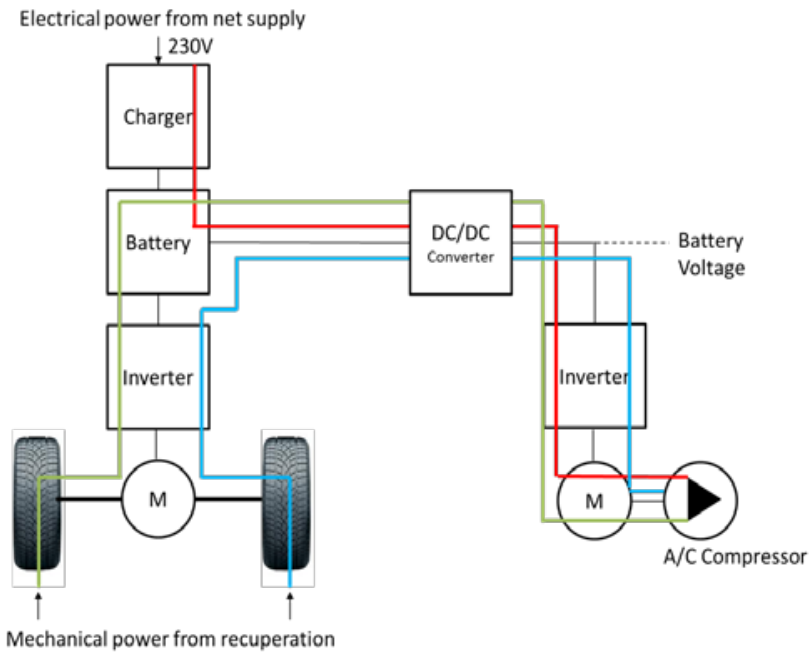

Fig. 7: Energy flux to feed the AC-compressor in a conventional electric vehicle (red: from supply net, green: from recuperated and stored energy, blue: from the wheel shaft without storing it in the battery)
Another step in terms of a high level of integration is the merging of the drive motor and further auxiliary units in one housing. Thereby, the overall efficiency of the vehicle drive is further increased by transmitting the power for the components directly from the drive shaft during recuperation.

An example is the integration of the air compressor in the driving motor. Figure 7 shows the energy flow from the net supply to the air conditioning compressor as it is today operated usual with an overall efficiency of about $60 \%$, regarding all the energy converters and storages located between the net and the compressor. In contrast, by coupling the compressor to the driven shaft the kinetic energy of the vehicle can be used with an efficiency nearly to $100 \%$ to feed the compressor, see figure 8 , which improves the total efficiency of an electric vehicle by a few percentage points.

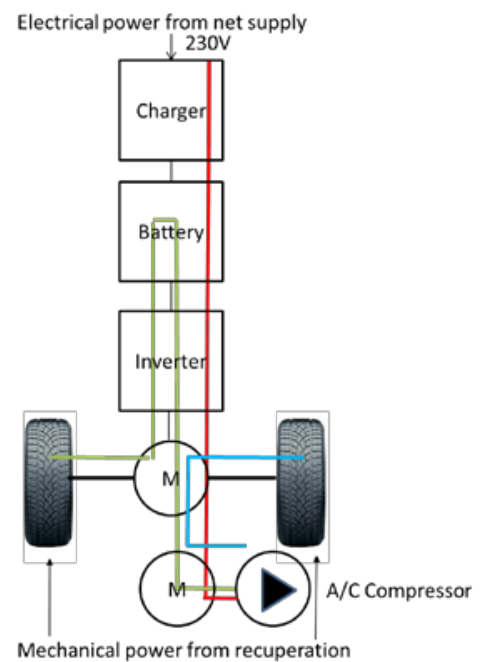

Fig. 8: Energy flux to feed the AC-compressor directly from the wheel shaft (blue line)

Further advantages of the integration are the saving of weight and component costs. Figure 9 shows a small urban electric vehicle test platform to install the motor with integrated AC-compressor.

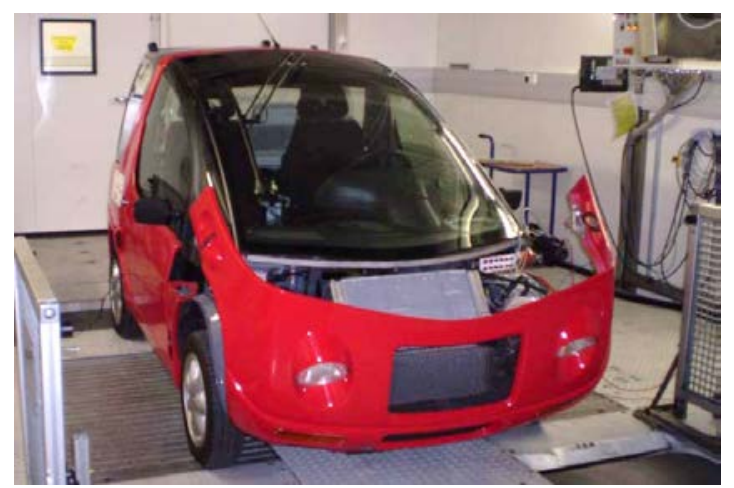

Fig. 9: Test platform to install a drive motor with integrated air conditioning compressor 


\section{E. Fuel Cell Thermal Management}

The integration of a high temperature PEM fuel cell in the drive train of the battery electric vehicles represents a promising solution to increase their electric range. This integration requires a development of a new system for the heating and cooling of the high temperature fuel cell. A potential concept has been investigated [5]. It is based on the thermal coupling of a high temperature fuel cell with a hydride storage tank (Fig. 10).

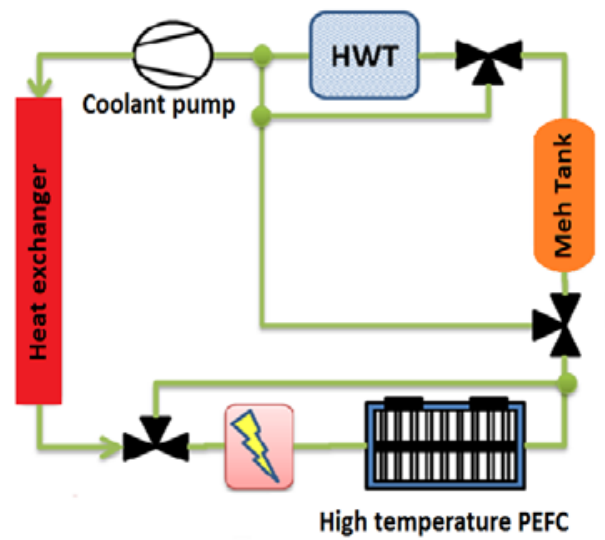

Fig. 10: Coupling of fuel-cells to the hybrid storage

In order to investigate the suitability of this system for electric vehicles, a complete vehicle simulation model is developed. It is used to minimize the fuel cell warm-up time and to optimize the fuel cell cooling, especially in the case of driving uphill or at very high ambient temperatures.

The fuel cell is modeled as a range extender for a small vehicle with a weight of $1155 \mathrm{~kg}$ (figure 12), with a power output of about $6 \mathrm{~kW}$, the range can be increased by about $38 \%$ in a real commute road cycle, see figure 11 .
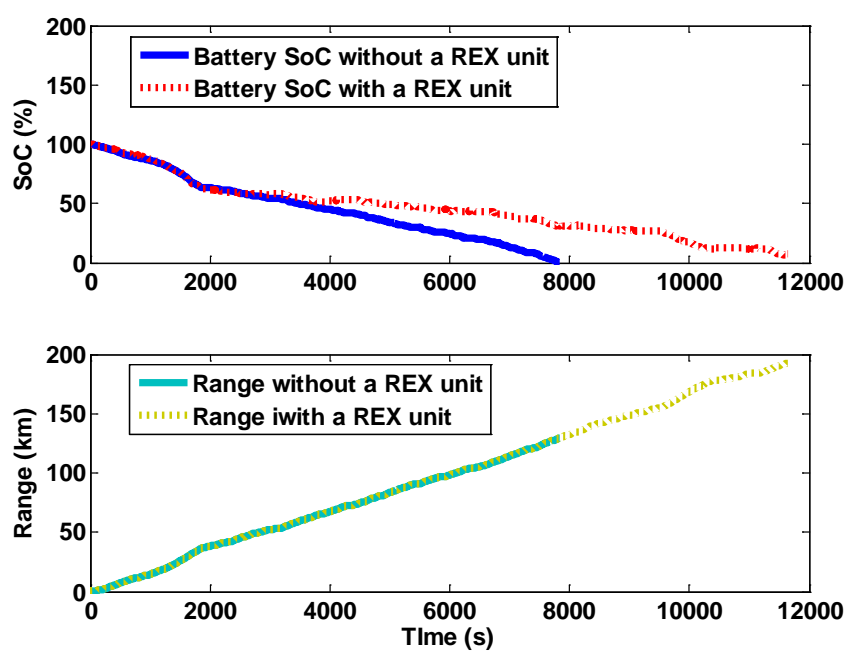

Fig. 11: Increasing the range of an electric vehicle by using a fuel cell as a range extender and the corresponding battery state of charge

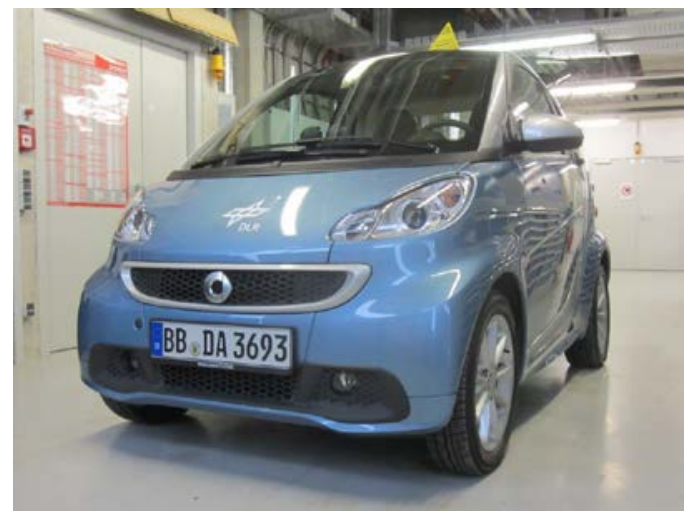

Fig. 12: Test platform for fuel cell range extender based on an electric Smart vehicle

\section{F. Thermochemical Storages}

To replace the electrical heater in figure 10 par example, another research focus in the area of thermal management is the development of energy-efficient heating systems, which are based on thermochemical storages. In the case of metal hydrides, the absorption and desorption of hydrogen are the basis for the storage of thermal energy.

Thermo-chemical storages are loss-free heat storages over long periods. In the case of metal hydrides (compounds containing metal or metalloid bonds to hydrogen), the absorption and desorption of hydrogen are the basis for the storage of thermal energy. When hydrogen is absorbed by the metal, heat is released because it is an exothermic reaction. The chemical reaction is reversible so that the process is endothermic und consuming heat, when hydrogen is released.

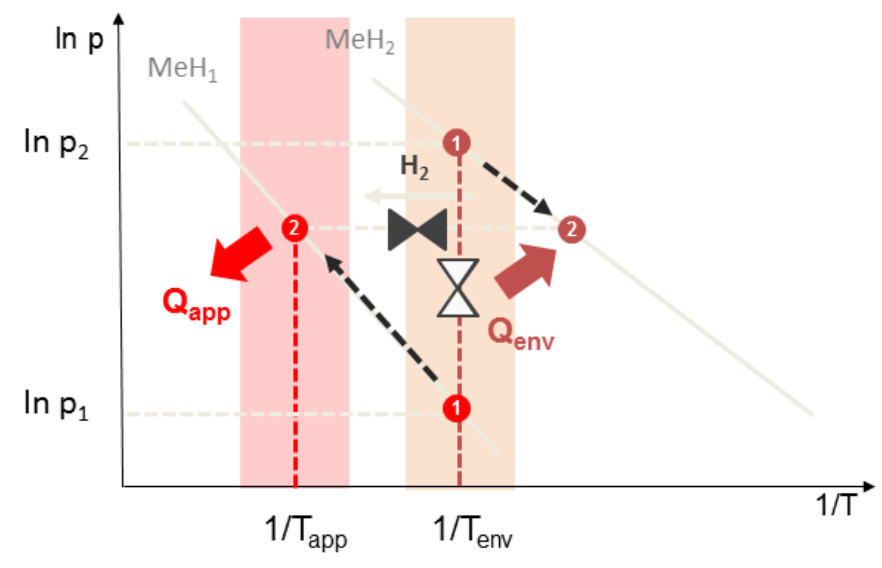

Fig. 13: Absorbing and release thermal energy by using two coupled metal hydrids 
Figure 13 shows the function of heat and coldness production. Two storages filled with two different metal hydrids in point 1 have the same temperature and different pressures, characterized by the closed valve in white color. If the two different metal hydrides $\mathrm{MeH}_{1}$ and $\mathrm{MeH}_{2}$, which are located on different pressure levels, are connected to each other as drawn by opening the black valves in the figure, one material delivers heat $\mathrm{Q}_{\text {app }}$ to an application and the other material absorbs heat $Q_{\text {env }}$ from the environment. Their temperature and pressure behavior moves from the points 1 two the points 2 .

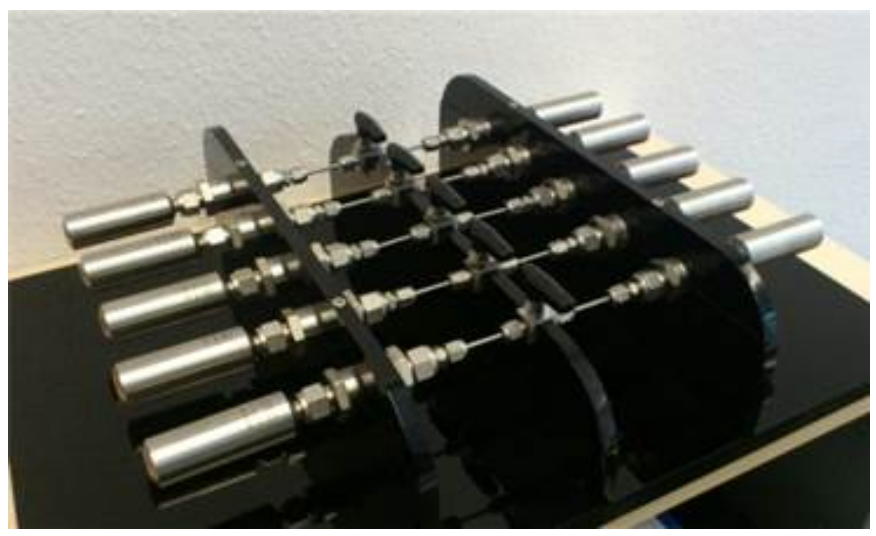

Fig. 14: Coupled thermochemical storages to produce heat and coldness at the same time

The energy density of such heat storages is significantly higher than the energy density of the sensible and latent heat storage. This difference can be expected up to factor 10 in comparison to sensible heat storage. The process of storing the chemical energy is reversible and it is possible to produce heat as well as coldness and store it without losses. Figure 14 shows a sample of five coupled thermochemical storages to demonstrate the different temperatures after opening the valve between two of them.

These novel systems are quieter and do not require chloroflourocarbons (CFC). CFC's, such as freon, have been linked to the destruction of stratospheric ozone that limits the amount of UV radiation reaching the earth's surface.

\section{G. Thermoelectric Energy Conversion}

Thermal energy can be converted into electrical energy with the thermoelectric energy conversion [1]. It is based on the thermoelectric effect, in which an electrical voltage is generated at the ends of an electrically conductive material, if the material at the two ends has different temperatures, see figure 15 .

The preferred materials have a good electrical conductivity and a poor thermal conductivity like bismuth telluride. This material is a semiconductor material and has a mechanical behavior similar to that of ceramic.

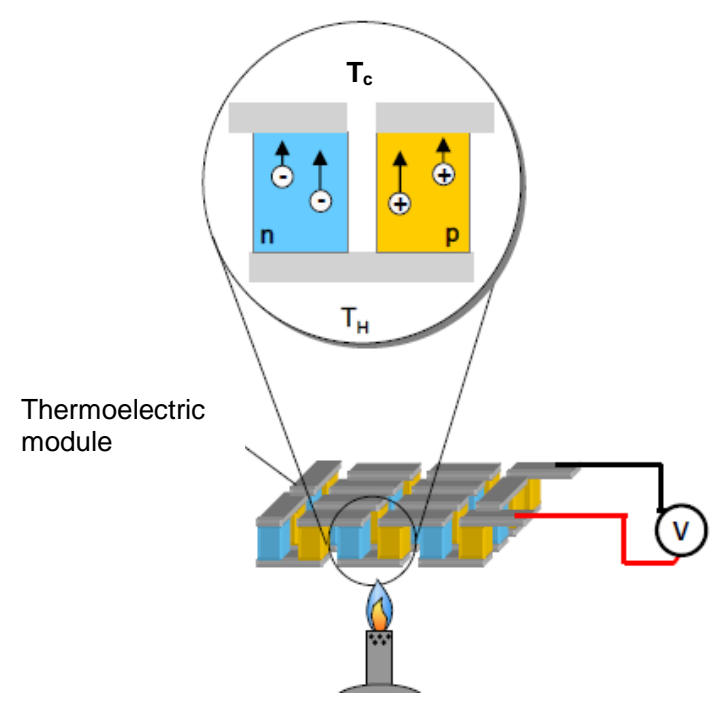

Fig. 15: Thermoelectric energy conversation based on Seebeckeffect, $T_{c}$ is the temperature at the cold side, $T_{h}$ the temperature at the hot side

A vehicle with an internal combustion engine with a thermoelectric generator has been demonstrated [2], see figure 16. This prototype of a thermal electric generator produced an electric power of $200 \mathrm{~W}$ at a vehicle speed of $130 \mathrm{~km} / \mathrm{h}$.

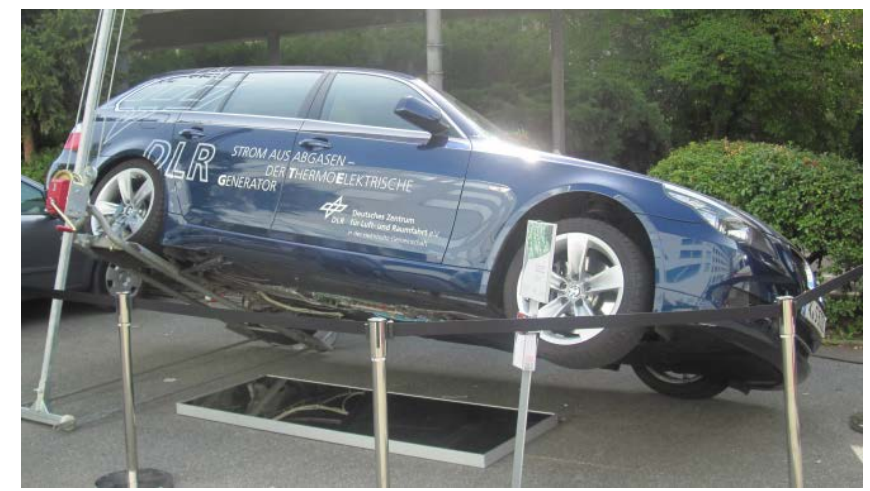

Fig.16: Test platform with thermoelectric generator in the exhaust system based on a BMW 5series

In the meantime, hybrid vehicles are available on the market. In the future, electric cars will be equipped with smaller or larger range extenders. Wherever heat is obtained at a high temperature, the thermoelectric brings a chance to increase the overall efficiency of the vehicle system. Figure 17 shows the test platform for thermoelectric energy conversion based on an Opel Ampera which has a range extender installed. 


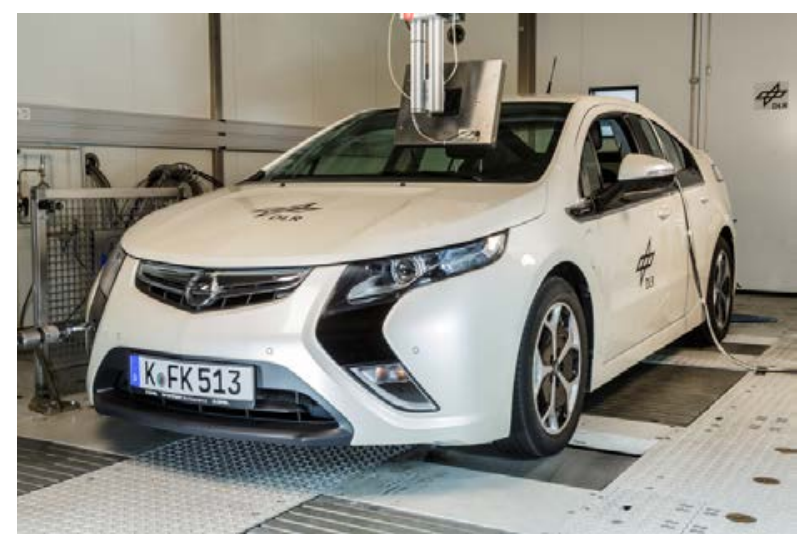

Fig. 17: Hybrid electric vehicle as test vehicle for thermoelectric generator

\section{CONCLUSION}

The paper gives summary of the current research activities in the field of thermal management at the German Aerospace Center. In this paper, the novel energy storage systems, the air-conditioning systems for the cabin, their challenges, design, simulation and possible integration concepts are presented and described. The focus of the paper is on the following selected topics: the high temperature PEM fuel cell used as a range extender to increase the range up to $140 \%$, the thermochemical storages, where the energy density in comparison to sensible heat storages is expected to make factor up to 10, the magneto caloric heat pumps with COP up to 5 and the usage of $\mathrm{CO}_{2}$ instead R1234yf refrigerant in the compressor expansion units based on the free piston linear generator concept. The presented topics are the basis for concepts of next generation vehicles.

\section{ACKNOWLEDGMENT}

The authors would like to thank the EVER 14 platform for publishing our wok.

Thermal management and the passenger comfort within the German Aerospace Center is part of the Next Generation Car and the Next Generation Train activities, where further institutes are working and have to get thanks: Institute of Technical Thermodynamic, Institute of Aerodynamics and Flow Technology, Institute of System Dynamics and Control.

The German Aerospace Center also cooperates with the Nanyang Technological University of Singapore in the field of thermal management and would like to acknowledge their support.

\section{REFERENCES}

[1] M. Schier and F. Rinderknecht, Innovation examples for ecological vehicles based on aerospace research, Eighth International Conference and Exhibition on Ecological Vehicles and Renewable Energies EVER 13, 23.-27.03.2013, MonteCarlo, Monaco

[2] C. Häfele and M. Schier and S. Hahn and T. Weiler and H. Friedrich, Experimental analysis of a test vehicle in terms of energetic potentials and retroactive effects on the vehicle system at the integration of thermoelectric generators, Thermoelectric Goes Automotive, pages 42-70. Expertverlag, Renningen. Thermoelectric Goes Automotive, 09.-10.12.2010, Berlin, Germany. ISBN 978-3-8169-3064-8

[3] F. Rinderknecht and F. Kock, A high efficient energy converter for a hybrid vehicle, Electric Vehicles Symposium EVS 26, 06.09.05.2012, Los Angeles, California

[4] F. Rinderknecht and H.G. Herzog, Adaptation and optimization of a linear generator for a hybrid vehicle concept, 25th World Battery, Hybrid and Fuel Cell Electric Vehicle Symposium and Exhibition, 05.-09.11.2010, Shenzhen, China

[5] P. Pfeifer and C. Wall et al., Thermal coupling of a high temperature PEM fuel cell with a complex hydride tank, International Journal of Hydrogen Energy 34, pp. 3457-3466, 2009

[6] J. Sarkar, Review on Cylce Modifications of Transcritical CO2 Refrigeration and Heat Pump Systems, Journal of Advanced Research in Mechanical Engineering 1, pp.22-29,2010

[7] V. Pérez-Garcia and J. Belman-Flores and J. Navarro-Esbrí and C Rubio-Maye, Comparative study of transcritical vapor compression configurations using CO2 as refrigeration mode base on simulation, Applied Thermal Engineering 51, pp. 10381046,2013

[8] Manh-Huong Phan and Seong-Cho Yu, Review of the Magnetocaloric Effect in Manganite Material, Journal of Magnetism and Magnetic Materials 308 (2007), 325-340

[9] K. A. Gschneidner and V. K. Pecharsky and A. O. Tsokol, Recent Developments in Magnetocaloric Materials, Rep. Prog. Phys. 68 (2005), 1479-1539

[10] F. C. Chen and R. W. Murphy and V. C. Mei and G. L. Chen, Thermodynamic Analysis of Four Magnetic Heat-pump Cyces, Journal of Engineering for Gas Turbines and Power 114 (1992), 71520 